\title{
Prognostic significance of preoperative aspartate aminotransferase to neutrophil ratio index in patients with hepatocellular carcinoma after hepatic resection
}

\author{
Fei $\mathrm{Ji}^{1,2,3, *}$, Shunjun Fu' ${ }^{1,2,3, *}$, Zhiyong Guo ${ }^{1,2,3 *}$, Hui Pang ${ }^{4}$, Dubo Chen ${ }^{5}$, Xiaoping \\ Wang ${ }^{1,2,3}$, Weiqiang $\mathrm{Ju}^{1,2,3}$, Dongping Wang ${ }^{1,2,3}$, Xiaoshun $\mathrm{He}^{1,2,3}$, Yunpeng Hua ${ }^{6}$ and \\ Baogang Peng ${ }^{6}$ \\ ${ }^{1}$ Organ Transplant Center, The First Affiliated Hospital, Sun Yat-sen University, Guangzhou, P. R. China \\ ${ }^{2}$ Guangdong Provincial Key Laboratory of Organ Donation and Transplant Immunology, Guangzhou, P. R. China \\ ${ }^{3}$ Guangdong Provincial International Cooperation Base of Science and Technology (Organ Transplantation), Guangzhou, P. \\ R. China \\ ${ }^{4}$ Department of Medical Records, The First Affiliated Hospital, Sun Yat-sen University, Guangzhou, P. R. China \\ ${ }^{5}$ Department of Laboratory Medicine, The First Affiliated Hospital, Sun Yat-sen University, Guangzhou, P. R. China \\ ${ }^{6}$ Department of Liver Surgery, The First Affiliated Hospital, Sun Yat-sen University, Guangzhou, P. R. China \\ * These authors have contributed equally to this work \\ Correspondence to: Baogang Peng, email: pengbaogang@medmail.com.cn \\ Yunpeng Hua, email: hyp0427@163.com \\ Xiaoshun He, email: gdtrc@163.com
}

Keywords: aspartate aminotransferase to neutrophil ratio index, systemic immune-inflammation, hepatocellular carcinoma, prognosis, biomarkers

Received: February 06, $2016 \quad$ Accepted: July 09, $2016 \quad$ Published: July 26, 2016

\section{ABSTRACT}

Objectives: Various inflammation-based prognostic scores have been associated with poor survival in patients with hepatocellular carcinoma (HCC), and neutrophils display important roles. However, few studies have illuminated the relationship between preoperative aspartate aminotransferase (AST) to neutrophil ratio index (ANRI) and poor prognosis of HCC. We aimed to clarify the prognostic value of ANRI and evaluate the ability of different inflammation-based prognostic scores such as ANRI, AST to lymphocyte ratio index (ALRI) ,AST to platelet count ratio index (APRI), neutrophil-lymphocyte ratio index (NLR), and platelet-lymphocyte ratio index (PLR).

Methods: Data were collected retrospectively from 303 patients who underwent curative resection for HCC. Preoperative ANRI, ALRI, APRI, NLR, PLR and clinicopathological variables were analyzed. Univariate, multivariate and Kaplan-Meier analyses were performed to identify the predictive value of the above factors for disease-free survival (DFS) and overall survival (OS).

Results: ANRI was correlated with presence of HBsAg, AST, presence of cirrhosis, tumor size, PVTT, cancer of the liver Italian program (CLIP) score, recurrence. Univariate analysis showed ANRI, ALRI, APRI,NLR, PLR were significantly associated with DFS and OS in HCC patients with curative resection. After multivariate analysis, ANRI was demonstrated to be superior to ALRI, APRI, NLR, PLR, which were independently correlated with DFS and OS. Survival analysis showed that preoperative ANRI > 7.8 predicted poor prognosis of patients with HCC after hepatectomy. preoperative ANRI also showed different prognostic value in various subgroups of HCC. Furthermore, the predictive range was expanded by the combination of ANRI and NLR.

Conclusions: preoperative ANRI is an independent effective predictor of prognosis for patients with HCC, higher levels of ANRI predict poorer outcomes and the combining ANRI and NLR increases the prognostic accuracy of testing. 


\section{INTRODUCTION}

Hepatocellular cancer (HCC) is one of the most common, and most aggressive malignancies, the third leading cause of cancer-related deaths worldwide $[1,2]$. Although the substantial increase in diagnostic techniques and surgery, the long-term prognosis of patients still remained far from satisfactory because almost $70 \%$ of patients develop recurrence or metastasis within 5 years after surgery [3-5]. The absence of an accurate and sensitive clinical parameter has a profound effect on $\mathrm{HCC}$ therapeutic practice. It is therefore very important and urgent to search for an effective preoperative biomarker to identify patients with a high risk of recurrence or metastases, and provide personalized therapy to improve the clinical outcomes.

Aspartate aminotransferase (AST) is an enzyme reflecting the damage of liver. As a routine liver function index, it is always used to evaluate the progression of diseases concerning liver [6-8]. Witjes [9] has also reported that the high serum levels of AST may indicate a poor survival in HCC patients. Another factor affects the prognosis is the host's inflammatory response to cancer and tumor-mediated systemic inflammation [10]. It is now believed that inflammatory cells in the tumor microenvironment have significant effects on tumor development [11-13]. The elevation of pretreatment neutrophil, monocyte or leucocyte count has been associated with poor survival in various cancers including HCC [15-17]. Emerging evidence indicates that neutrophil may reflect the patients' inflammatory status and downregulate host cellular immunity against cancer, thereby affecting the prognosis [14].

To our knowledge, the preoperative AST to neutrophil ratio index (ANRI) has not been conducted to predict the tumor recurrence and survival after curative resection for $\mathrm{HCC}$, though preoperative AST to lymphocyte ratio index (ALRI) and AST to platelet count ratio index (APRI) have been demonstrated to be associated to the poor survival [17-19]. The aim of this study is to clarify the prognostic value of ANRI in $\mathrm{HCC}$, we also evaluate the prognostic ability of different inflammation-based prognostic scores such as ANRI, ALRI, APRI, neutrophil-lymphocyte ratio (NLR), and platelet-lymphocyte ratio (PLR) to determine whether the ANRI could be a useful marker in predicting patients' outcomes.

\section{RESULTS}

\section{Patient and tumor characteristics}

The study included 271 male patients $(89.4 \%)$ and 32 female patients $(10.6 \%)$. The mean age was 50 years (range 21-79 years). A total of 222 patients $(73.3 \%$ ) developed recurrence and 192 patients (63.4\%) died during follow up. Hepatitis B surface antigen (HBsAg) was positive in 266 patients (87.8\%) and cirrhosis in 237 (78.2\%) patients. Increased AFP levels ( $\geq 200 \mu \mathrm{g} / \mathrm{L})$ were observed in 171 patients $(56.4 \%)$, and 88 patients $(29.0 \%)$ had multiple tumor masses. Mean tumor size was $80.7 \mathrm{~mm}$ (range 15-300 mm) in greatest diameter, and $198(65.3 \%)$ patients had tumor $\geq 50 \mathrm{~mm}$ in diameter. According to the Edmonson-Steiner stage [20] of tumor differentiation, $235(77.6 \%)$ patients were in stages I-II and $68(22.4 \%)$ patients were in stages III-IV. Likewise, according to TNM classification [21], $175(57.8 \%)$ patients were in TNM stage I and 128 (42.2\%) patients in TNM stage IIIII (Table 1).

\section{Determination of cut-off value}

Using 5-year overall survival rate as an endpoint, stratification of NLR, PLR, ALRI, APRI, ANRI was calculated by ROC curve analyses. The results showed the Area Under the Curve (AUC) of NLR, PLR, ALRI, APRI, ANRI was $0.580,0.588,0.656,0.629,0.641$ and the optimal cut-off value was $2,115,20,1.68,7.8$, corresponding to the maximum joint sensitivity and specificity, respectively. (Figure 1).

\section{Correlation between ANRI and clinicopathologic characteristics in $\mathrm{HCC}$}

The relationship between preoperative ANRI and clinicopathological variables of patients with HCC was investigated, and the data showed that preoperative ANRI was correlated with HBsAg $(P=0.003)$, AST $(P$ $<0.001)$, presence of cirrhosis $(P=0.001)$, tumor size $(P=0.006)$, PVTT $(P=0.045)$, cancer of the liver Italian program (CLIP) score $(P=0.001)$, recurrence $(P<0.001)$. the c-index between ANRI and CLIP were 0.70, which showed a concordance with the CLIP. Nevertheless, there were no significance between preoperative ANRI and other clinicopathological parameters such as age, gender, AFP, tumor number, TNM, tumor differentiation and complication (all $P>0.05$, Table 1).

\section{Independent prognostic factors for $\mathrm{HCC}$}

In order to further identify the risk factors related to postoperative DFS and OS, ANRI and the clinicopathological parameters were evaluated by univariate analysis and the Cox regression model. The results revealed that preoperative ANRI $(P<0.001)$, HBsAg $(P=0.02)$, AFP $(P<0.001)$, AST $(P<0.001)$, Neutrophil $(P=0.018)$, CLIP score $(P<0.001)$, TNM $(P$ $=0.001)$, PVTT $(P<0.001)$, tumor number $(P<0.001)$, 
Table 1: Correlation between preoperative ANRI and clinicopathologic characteristics in HCC

\begin{tabular}{|c|c|c|c|c|c|c|}
\hline \multirow[t]{2}{*}{ Variables } & \multirow[t]{2}{*}{ Cases } & \multicolumn{2}{|c|}{ ANRI } & \multirow{2}{*}{$X^{2}$} & \multirow{2}{*}{$r$} & \multirow{2}{*}{$P$ value } \\
\hline & & $\leq 7.8$ & $>7.8$ & & & \\
\hline \multicolumn{7}{|l|}{ Age(yrs) } \\
\hline$\geq 60$ & 73 & $24(32.9 \%)$ & $49(67.1 \%)$ & \multirow{2}{*}{0.287} & & \multirow{2}{*}{0.592} \\
\hline$<60$ & 230 & $68(29.6 \%)$ & $162(70.4 \%)$ & & & \\
\hline \multicolumn{7}{|l|}{ Gender } \\
\hline Male & 271 & $80(29.5 \%)$ & $191(70.5 \%)$ & \multirow{2}{*}{0.862} & & \multirow{2}{*}{0.353} \\
\hline Female & 32 & $12(37.5 \%)$ & $20(62.5 \%)$ & & & \\
\hline \multicolumn{7}{|c|}{ HCC family history } \\
\hline Yes & 21 & $9(42.9 \%)$ & $12(57.1 \%)$ & \multirow{2}{*}{1.666} & & \multirow{2}{*}{0.197} \\
\hline No & 282 & $83(29.4 \%)$ & $199(70.6 \%)$ & & & \\
\hline \multicolumn{7}{|l|}{$\mathrm{HBsAg}$} \\
\hline Positive & 266 & $73(27.4 \%)$ & $193(72.6 \%)$ & \multirow{2}{*}{8.781} & \multirow{2}{*}{0.170} & \multirow{2}{*}{0.003} \\
\hline Negative & 37 & $19(51.4 \%)$ & $18(48.6 \%)$ & & & \\
\hline \multicolumn{7}{|l|}{ AST(U/L) } \\
\hline$<80$ & 249 & $92(36.9 \%)$ & $157(63.1 \%)$ & \multirow{2}{*}{28.651} & & \\
\hline$\geq 80$ & 54 & $0(0 \%)$ & $54(100 \%)$ & & 0.308 & $<0.001$ \\
\hline $\mathrm{TBIL}(\mu \mathrm{mc}$ & & & & & & \\
\hline$<34.2$ & 277 & $88(31.8 \%)$ & $189(68.2 \%)$ & & & \\
\hline$\geq 34.2$ & 26 & $4(15.4 \%)$ & $22(84.6 \%)$ & 3.018 & & 0.082 \\
\hline $\operatorname{PLT}\left(\times 10^{9}\right)$ & & & & & & \\
\hline$\geq 100$ & 275 & $87(31.6 \%)$ & $188(68.4 \%)$ & & & \\
\hline$<100$ & 28 & $5(17.9 \%)$ & $23(82.1 \%)$ & 2.282 & & 0.131 \\
\hline Cirrhosis & & & & & & \\
\hline Yes & 237 & $61(25.7 \%)$ & $176(74.3 \%)$ & 11006 & 0101 & 0001 \\
\hline No & 66 & $31(47.0 \%)$ & $35(53.0 \%)$ & 11.000 & 0.191 & 0.001 \\
\hline $\operatorname{AFP}(\mu \mathrm{g} / \mathrm{L}$ & & & & & & \\
\hline$\geq 200$ & 171 & $48(28.1 \%)$ & $123(71.9 \%)$ & & & 0323 \\
\hline$<200$ & 132 & $44(33.3 \%)$ & $88(66.7 \%)$ & 0.976 & & 0.323 \\
\hline Tumor siz & & & & & & \\
\hline$>5$ & 198 & $51(25.8 \%)$ & $147(74.2 \%)$ & & & \\
\hline$\leq 5$ & 105 & $41(39.0 \%)$ & $64(61.0 \%)$ & 5.732 & 0.156 & 0.017 \\
\hline Tumor nur & & & & & & \\
\hline Single & 215 & $71(33.0 \%)$ & $144(67.0 \%)$ & & & 0115 \\
\hline Multiple & 88 & $21(23.9 \%)$ & $67(76.1 \%)$ & $2.4 / 8$ & & 0.115 \\
\hline TNM & & & & & & \\
\hline $\mathrm{I}$ & 175 & $59(33.7 \%)$ & $116(66.3 \%)$ & & & 0120 \\
\hline II-III & 128 & $33(25.8 \%)$ & $95(74.2 \%)$ & 2.200 & & 0.138 \\
\hline Differentic & & & & & & \\
\hline I-II & 235 & $75(31.9 \%)$ & $160(68.1 \%)$ & & & 0275 \\
\hline III-IV & 68 & $17(25.0 \%)$ & $51(75.0 \%)$ & 1.193 & & 0.275 \\
\hline PVTT & & & & & & \\
\hline Yes & 53 & $10(18.9 \%)$ & $43(81.1 \%)$ & 4014 & 0115 & 0045 \\
\hline No & 250 & $82(32.8 \%)$ & $168(67.2 \%)$ & 4.014 & 0.113 & 0.045 \\
\hline CLIP scor & & & & & & \\
\hline $0-2$ & 217 & $78(35.9 \%)$ & $139(64.1 \%)$ & 11265 & 0102 & 0001 \\
\hline $3-5$ & 86 & $14(16.3 \%)$ & $72(83.7 \%)$ & 11.265 & 0.193 & 0.001 \\
\hline Recurrenc & & & & & & \\
\hline Yes & 222 & $52(23.4 \%)$ & $170(76.6 \%)$ & & & \\
\hline No & 81 & $40(49.4 \%)$ & $41(50.6 \%)$ & 18.915 & 0.250 & $<0.001$ \\
\hline
\end{tabular}




\begin{tabular}{|l|l|l|l|l|l|l|}
\hline Complication & & & & & & \\
\cline { 1 - 6 } No & 258 & $82(31.8 \%)$ & $176(68.2 \%)$ & \multirow{2}{*}{1.656} & & \multirow{2}{*}{0.198} \\
\hline Yes & 45 & $10(22.2 \%)$ & $35(77.8 \%)$ & & & \\
\hline
\end{tabular}

HBsAg: hepatitis B surface antigen; AST: aspartate aminotransferase ratio index; TBIL: total bilirubin; PLT: platelet; AFP:alpha-fetoprotein; TNM: Tumor Node Metastasis; PVTT: portal vein tumor thrombus; CLIP: cancer of the liver Italian program; ANRI, Aspartate aminotransferase/ neutrophil count ratio index.

Table 2: Prognostic factors for DFS and OS by univariate analysis

\begin{tabular}{|c|c|c|c|c|c|c|c|c|c|}
\hline \multirow{2}{*}{ Variables } & \multirow{2}{*}{$n$} & \multicolumn{3}{|l|}{ DFS } & \multirow{2}{*}{$P$} & \multicolumn{3}{|l|}{ OS } & \multirow{2}{*}{$P$} \\
\hline & & 1-yr & 3-yrs & 5-yrs & & $1-y r$ & 3-yrs & 5-yrs & \\
\hline \multicolumn{10}{|l|}{ Gender } \\
\hline Male & 271 & $43.2 \%$ & $29.9 \%$ & $26.0 \%$ & \multirow{2}{*}{0.154} & $70.1 \%$ & $44.6 \%$ & $36.8 \%$ & \multirow{2}{*}{0.032} \\
\hline Female & 32 & $56.3 \%$ & $37.5 \%$ & $37.5 \%$ & & $81.3 \%$ & $59.4 \%$ & $56.3 \%$ & \\
\hline \multicolumn{10}{|l|}{ Age(yrs) } \\
\hline$<60$ & 230 & $43.9 \%$ & $32.2 \%$ & $29.5 \%$ & \multirow{2}{*}{0.457} & $70.9 \%$ & $47.4 \%$ & $39.9 \%$ & \multirow{2}{*}{0.430} \\
\hline$\geq 60$ & 73 & $46.6 \%$ & $26.0 \%$ & $20.0 \%$ & & $72.6 \%$ & $42.5 \%$ & $35.5 \%$ & \\
\hline \multicolumn{10}{|l|}{$\mathrm{HBsAg}$} \\
\hline Positive & 266 & $41.4 \%$ & $28.9 \%$ & $25.0 \%$ & \multirow{2}{*}{0.02} & $69.2 \%$ & $44.4 \%$ & $37.5 \%$ & \multirow{2}{*}{0.075} \\
\hline Negative & 37 & $67.6 \%$ & $43.2 \%$ & $43.2 \%$ & & $86.5 \%$ & $59.5 \%$ & $48.6 \%$ & \\
\hline \multicolumn{10}{|l|}{$\operatorname{AFP}(\mu \mathrm{g} / \mathrm{L})$} \\
\hline$<200$ & 132 & $54.5 \%$ & $38.6 \%$ & $36.3 \%$ & \multirow{2}{*}{$<0.001$} & $78.8 \%$ & $53.0 \%$ & $46.2 \%$ & \multirow{2}{*}{0.006} \\
\hline$\geq 200$ & 171 & $36.8 \%$ & $24.6 \%$ & $20.3 \%$ & & $65.5 \%$ & $40.9 \%$ & $33.2 \%$ & \\
\hline \multicolumn{10}{|l|}{$\mathrm{Hb}(\mathrm{g} / \mathrm{L})$} \\
\hline$\leq 120$ & 48 & $31.2 \%$ & $16.7 \%$ & $16.7 \%$ & \multirow{2}{*}{0.052} & $68.7 \%$ & $33.3 \%$ & $29.2 \%$ & \multirow{2}{*}{0.222} \\
\hline$>120$ & 255 & $47.1 \%$ & $34.1 \%$ & $29.2 \%$ & & $71.8 \%$ & $48.6 \%$ & $40.7 \%$ & \\
\hline \multicolumn{10}{|l|}{$\mathrm{WBC}\left(\times 10^{9}\right)$} \\
\hline$<10$ & 271 & $45.4 \%$ & $31.4 \%$ & $27.5 \%$ & \multirow{2}{*}{0.588} & $72.3 \%$ & $46.1 \%$ & $39.0 \%$ & \multirow{2}{*}{0.977} \\
\hline$\geq 10$ & 32 & $37.5 \%$ & $25.0 \%$ & $25.0 \%$ & & $62.5 \%$ & $46.9 \%$ & $37.5 \%$ & \\
\hline \multicolumn{10}{|l|}{ AST(U/L) } \\
\hline$<80$ & 150 & $57.3 \%$ & $41.3 \%$ & $37.8 \%$ & \multirow{2}{*}{$<0.001$} & $83.3 \%$ & $58.7 \%$ & $50.6 \%$ & \multirow{2}{*}{$<0.001$} \\
\hline$\geq 80$ & 153 & $32.0 \%$ & $20.3 \%$ & $16.9 \%$ & & $59.5 \%$ & $34.0 \%$ & $27.4 \%$ & \\
\hline Neutrophil( & & & & & & & & & \\
\hline$<3.94$ & 168 & $49.4 \%$ & $35.1 \%$ & $30.8 \%$ & 0018 & $78.6 \%$ & $51.2 \%$ & $44.0 \%$ & 0032 \\
\hline$\geq 3.94$ & 135 & $38.5 \%$ & $25.2 \%$ & $22.8 \%$ & 0.018 & $62.2 \%$ & $40.0 \%$ & $32.6 \%$ & 0.032 \\
\hline CLIP score & & & & & & & & & \\
\hline $0-2$ & 217 & $55.3 \%$ & $39.2 \%$ & $34.4 \%$ & & $80.6 \%$ & $56.2 \%$ & $48.8 \%$ & \\
\hline $3-5$ & 86 & $17.4 \%$ & $9.3 \%$ & $9.3 \%$ & $<0.001$ & $47.7 \%$ & $20.9 \%$ & $13.4 \%$ & $<0.001$ \\
\hline TNM & & & & & & & & & \\
\hline $\mathrm{I}$ & 175 & $52.0 \%$ & $36.6 \%$ & $32.4 \%$ & 0001 & $77.7 \%$ & $54.3 \%$ & $47.3 \%$ & $<00001$ \\
\hline II-III & 128 & $34.4 \%$ & $22.7 \%$ & $20.1 \%$ & 0.001 & $62.5 \%$ & $35.2 \%$ & $27.3 \%$ & $<0.001$ \\
\hline Cirrhosis & & & & & & & & & \\
\hline No & 66 & $40.9 \%$ & $27.3 \%$ & $24.2 \%$ & 0454 & $75.8 \%$ & $45.5 \%$ & $37.9 \%$ & 0803 \\
\hline Yes & 237 & $45.6 \%$ & $31.6 \%$ & $28.1 \%$ & 0.454 & $70.0 \%$ & $46.4 \%$ & $39.2 \%$ & 0.803 \\
\hline
\end{tabular}

HBsAg: hepatitis B surface antigen; AFP:alpha-fetoprotein; Hb: hemoglobin; WBC: white blood cell; AST: aspartate aminotransferase ratio index; CLIP: cancer of the liver Italian program; TNM: Tumor Node Metastasis.

tumor size $(P<0.001)$, tumor differentiation $(P=0.003)$, and intraoperative blood loss $(P<0.001)$, NLR $(P<$ $0.001)$, APRI $(P<0.001)$, ALRI $(P<0.001)$, PLR $(P=$ $0.025)$ were responsible for the DFS of HCC patients. Similarly, the significant predictors of OS in patients with $\mathrm{HCC}$ after resection were preoperative ANRI $(P<$
$0.001)$, gender $(P=0.032)$, AFP $(P=0.006)$, AST $(P<$ $0.001)$, Neutrophil $(P=0.032)$, CLIP score $(P<0.001)$, TNM $(P<0.001)$, PVTT $(P<0.001)$, tumor number $(P$ $<0.001)$, tumor size $(P<0.001)$, tumor differentiation $(P$ $=0.006)$, intraoperative blood loss $(P<0.001), \operatorname{NLR}(P$ $<0.001)$, APRI $(P<0.001)$, ALRI $(P<0.001), \operatorname{PLR}(P$ 
Table 3: Prognostic factors for DFS and OS by univariate analysis (to be continued)

\begin{tabular}{|c|c|c|c|c|c|c|c|c|c|}
\hline \multicolumn{10}{|l|}{ PVTT } \\
\hline No & 250 & $51.6 \%$ & $35.2 \%$ & $31.4 \%$ & \multirow{2}{*}{$<0.001$} & $78.8 \%$ & $52.0 \%$ & $44.7 \%$ & \multirow{2}{*}{$<0.001$} \\
\hline Yes & 53 & $11.3 \%$ & $9.4 \%$ & $7.5 \%$ & & $35.8 \%$ & $18.9 \%$ & $11.0 \%$ & \\
\hline \multicolumn{10}{|c|}{ Tumor number } \\
\hline Single & 215 & $53.5 \%$ & $38.1 \%$ & $33.9 \%$ & \multirow{2}{*}{$<0.001$} & $77.0 \%$ & $54.9 \%$ & $48.6 \%$ & \multirow{2}{*}{$<0.001$} \\
\hline Multiple & 88 & $22.7 \%$ & $12.4 \%$ & $10.6 \%$ & & $56.8 \%$ & $26.3 \%$ & $16.7 \%$ & \\
\hline \multicolumn{10}{|c|}{ Tumor size(cm) } \\
\hline$<5$ & 105 & $68.6 \%$ & $51.4 \%$ & $43.5 \%$ & \multirow{2}{*}{$<0.001$} & $88.6 \%$ & $68.6 \%$ & $60.0 \%$ & \multirow{2}{*}{$<0.001$} \\
\hline$\geq 5$ & 198 & $31.8 \%$ & $19.7 \%$ & $18.7 \%$ & & $62.1 \%$ & $34.3 \%$ & $27.7 \%$ & \\
\hline \multicolumn{10}{|c|}{ Complication } \\
\hline No & 258 & $44.6 \%$ & $32.2 \%$ & $28.1 \%$ & \multirow{2}{*}{0.424} & $72.5 \%$ & $46.5 \%$ & $40.2 \%$ & \multirow{2}{*}{0.361} \\
\hline Yes & 45 & $44.4 \%$ & $22.2 \%$ & $22.2 \%$ & & $64.4 \%$ & $44.4 \%$ & $30.8 \%$ & \\
\hline \multicolumn{10}{|c|}{ Tumor differentiation } \\
\hline I-II & 235 & $48.5 \%$ & $34.0 \%$ & $30.5 \%$ & \multirow{2}{*}{0.003} & $73.6 \%$ & $48.9 \%$ & $42.9 \%$ & \multirow{2}{*}{0.006} \\
\hline III-IV & 68 & $30.9 \%$ & $19.1 \%$ & $16.2 \%$ & & $63.2 \%$ & $36.8 \%$ & $24.8 \%$ & \\
\hline \multicolumn{10}{|c|}{ Resection margin $(\mathrm{cm})$} \\
\hline$<2$ & 171 & $42.1 \%$ & $26.9 \%$ & $23.2 \%$ & \multirow{2}{*}{0.186} & $70.8 \%$ & $41.5 \%$ & $34.4 \%$ & \multirow{2}{*}{0.069} \\
\hline$\geq 2$ & 131 & $47.3 \%$ & $35.1 \%$ & $31.9 \%$ & & $71.8 \%$ & $51.9 \%$ & $44.2 \%$ & \\
\hline \multicolumn{10}{|c|}{ Intraoperative blood loss(ml) } \\
\hline$\leq 1000$ & 237 & $48.9 \%$ & $34.6 \%$ & $31.0 \%$ & \multirow{2}{*}{$<0.001$} & $76.4 \%$ & $50.6 \%$ & $43.8 \%$ & \multirow{2}{*}{$<0.001$} \\
\hline$>1000$ & 66 & $28.8 \%$ & $16.7 \%$ & $13.6 \%$ & & $53.0 \%$ & $30.3 \%$ & $21.0 \%$ & \\
\hline \multicolumn{10}{|l|}{ ANRI } \\
\hline$\leq 7.8$ & 92 & $59.8 \%$ & $46.7 \%$ & $43.3 \%$ & \multirow{2}{*}{$<0.001$} & $81.5 \%$ & $62.0 \%$ & $55.4 \%$ & \multirow{2}{*}{$<0.001$} \\
\hline$>7.8$ & 211 & $37.9 \%$ & $23.7 \%$ & $20.2 \%$ & & $66.8 \%$ & $39.3 \%$ & $31.6 \%$ & \\
\hline \multicolumn{10}{|l|}{ NLR } \\
\hline$\leq 2$ & 223 & $48.9 \%$ & $35.9 \%$ & $31.7 \%$ & \multirow{2}{*}{$<0.001$} & $75.3 \%$ & $53.4 \%$ & $45.7 \%$ & -0001 \\
\hline$>2$ & 80 & $32.5 \%$ & $16.2 \%$ & $14.9 \%$ & & $60.0 \%$ & $26.3 \%$ & $20.0 \%$ & \\
\hline APRI & & & & & & & & & \\
\hline$\leq 1.68$ & 104 & $57.7 \%$ & $41.3 \%$ & $38.3 \%$ & & $82.7 \%$ & $59.6 \%$ & $52.9 \%$ & $<0.001$ \\
\hline$>1.68$ & 199 & $37.7 \%$ & $25.1 \%$ & $21.4 \%$ & 0.001 & $65.3 \%$ & $39.2 \%$ & $31.5 \%$ & \\
\hline PLR & & & & & & & & & \\
\hline$\leq 115$ & 173 & $50.3 \%$ & $35.8 \%$ & $30.5 \%$ & 0025 & $75.7 \%$ & $35.0 \%$ & $45.6 \%$ & 0005 \\
\hline$>115$ & 130 & $36.9 \%$ & $23.8 \%$ & $23.1 \%$ & $0.0<\mathrm{J}$ & $65.4 \%$ & $35.4 \%$ & $29.9 \%$ & 0.000 \\
\hline ALRI & & & & & & & & & \\
\hline$\leq 20$ & 135 & $57.0 \%$ & $42.2 \%$ & $38.5 \%$ & & $81.5 \%$ & $60.7 \%$ & $54.1 \%$ & \\
\hline$>20$ & 168 & $34.5 \%$ & $21.4 \%$ & $18.0 \%$ & 0.001 & $63.1 \%$ & $34.5 \%$ & $26.5 \%$ & 0.001 \\
\hline
\end{tabular}

PVTT: portal vein tumor thrombus; ANRI, Aspartate aminotransferase/ neutrophil count ratio index; NLR: neutrophil/ lymphocyte ratio index; APRI: aspartate aminotransferase/ platelet count ratio index; PLR: platelet/lymphocyte ratio index; ALRI: aspartate aminotransferase/Lymphocyte count ratio index. 
Table 4: Independent prognostic factors for DFS and OS by the multivariate Cox proportional hazards regression model

\begin{tabular}{|l|l|l|l|l|l|l|}
\hline \multirow{2}{*}{ Variables } & \multicolumn{3}{c|}{ DFS } & \multicolumn{3}{c|}{ OS } \\
\cline { 2 - 7 } & HR & $\mathbf{9 5 \%}$ CI & $\boldsymbol{P}$ & HR & $\mathbf{9 5 \%}$ CI & $\boldsymbol{P}$ \\
\hline AFP & 1.393 & $1.056-1.836$ & 0.019 & & & \\
\hline Tumor Size & 1.579 & $1.144-2.179$ & 0.005 & & & \\
\hline Tumor number & 0.581 & $0.435-0.777$ & $<0.001$ & 0.650 & $0.465-0.910$ & 0.012 \\
\hline PVTT & 2.119 & $1.506-2.982$ & $<0.001$ & 2.156 & $1.507-3.085$ & $<0.001$ \\
\hline ANRI & 1.747 & $1.222-2.498$ & 0.002 & 1.617 & $1.108-2.358$ & 0.013 \\
\hline Neutrophil & 1.490 & $1.108-2.003$ & 0.008 & & & \\
\hline CLIP score & & & & 1.459 & $1.015-2.097$ & 0.042 \\
\hline AST & & & & 1.582 & $1.094-2.287$ & 0.015 \\
\hline NLR & & & & 1.724 & $1.241-2.394$ & 0.001 \\
\hline
\end{tabular}

HR, hazard ratio; CI, confidence interval; AFP, Alpha-fetoprotein; PVTT, portal vein tumor thrombi; ANRI, Aspartate aminotransferase/neutrophil count ratio index; CLIP: cancer of the liver Italian program; AST: aspartate aminotransferase ratio index; NLR: neutrophil/lymphocyte ratio index.

$=0.005$ ) (Table 2 and 3). After multivariate analysis, we found that AFP, tumor size, tumor number, PVTT, ANRI and Neutrophil were significant independent predictors of DFS (all $P<0.05$ ), while tumor number, PVTT, ANRI, CLIP score, AST and NLR were significant independent predictors of OS (all $P<0.05$ ) (Table 4).

\section{Overall and disease free survival rates according to ANRI}

To determine the ability of ANRI to predict OS and DFS, the 303 HCC patients were divided into two groups: the ANRI $\leq 7.8$ group $(n=92)$ and the ANRI $>7.8$ group

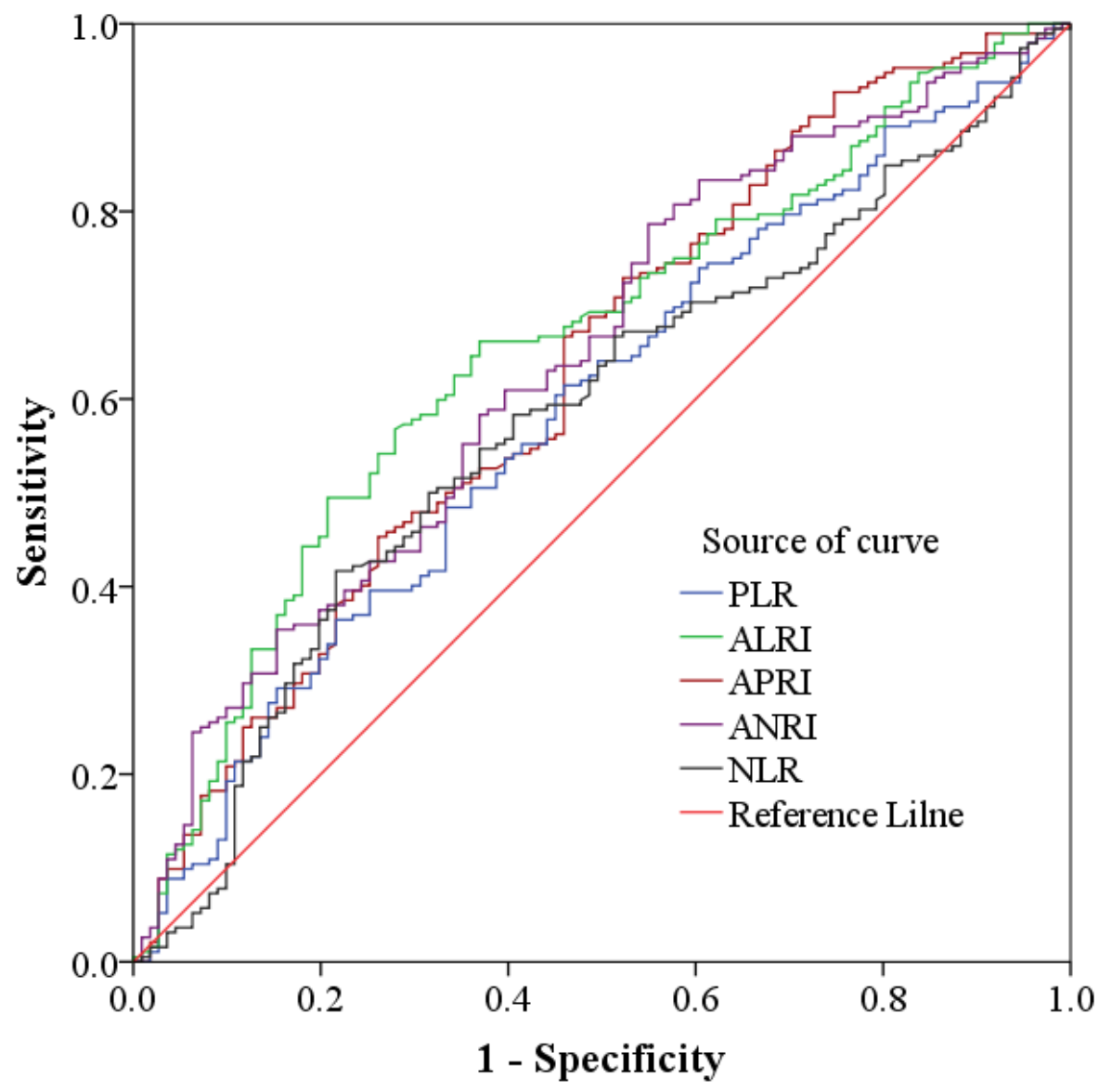

Figure 1: Determination of the cut-off value for NLR, PLR, ALRI, APRI, ANRI in HCC patients with hepatic resection. 
( $n=211)$. Using the Kaplan-Meier method to analyze patient survival, The data showed that the 1-, 3- and 5-year DFS rates of the ANRI $\leq 7.8$ group were significantly higher than those of the ANRI > 7.8 group (59.8\%, 46.7\% and $43.3 \%$ vs $40.8 \%, 23.7 \%$ and $20.2 \%$, respectively, $P<0.001$ ) (Figure 2A), while the 1-, 3- and 5-year OS rates of the ANRI $\leq 7.8$ group were also markedly higher than those of the ANRI $>7.8$ group $(81.5 \%, 62.0 \%$ and $55.4 \%$ vs $70.6 \%, 39.3 \%$ and $31.6 \%$, respectively, $P<$ 0.001 ) (Figure 2B). Therefore, our research displayed that preoperative ANRI > 7.8 levels were correlated with a poor survival.

\section{Prognostic values of preoperative ANRI in different HCC subgroups}

The research above verified that preoperative ANRI was an independent prognostic factor and significantly correlated with DFS and OS of HCC patients. We next assessed the prognostic value of preoperative ANRI in different subgroups of HCC patients. The results showed that preoperative ANRI was a prognostic indicator for DFS ( 69.5\%, 54.2\%, 50.7\% vs 48.3\%, 27.6\%, 23.1\%, $P$ $<0.001$, respectively) and OS $(88.1 \%, 74.6 \%, 66.1 \% v s$ $77.6 \%, 44.0 \%, 37.7 \%, P<0.001$, respectively) in patients with TNM stage of I (Figure 3A, 3B). In addition, in the subgroup of tumor size $>5 \mathrm{~cm}$, preoperative ANRI $>7.8$ also appeared noticeable prognostic value in predicting poorer DFS $(47.1 \%, 37.3 \%, 35.3 \%$ vs $26.5 \%, 13.6 \%$, $12.9 \%, P=0.003$, respectively) and OS ( $72.5 \%, 49.0 \%$, $43.1 \%$ vs $58.5 \%, 29.3 \%, 22.2 \%, P=0.003$, respectively)

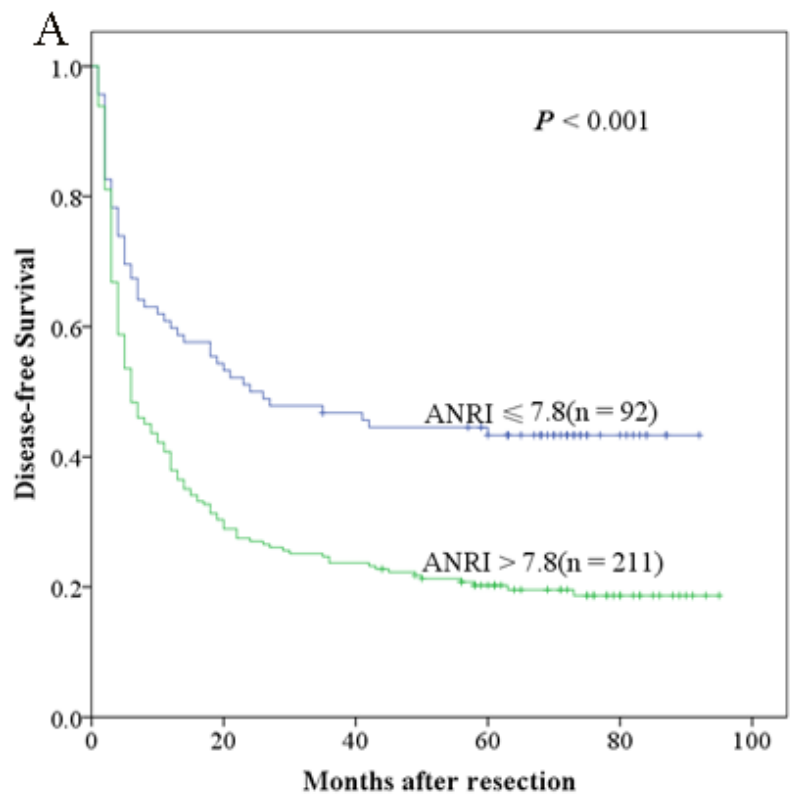

(Figure 3C, 3D), and this prognostic value of DFS (64.6\%, $51.2 \%, 47.4 \%$ vs $45.2 \%, 27.4 \%, 23.7 \%, P<0.001$, respectively) and OS ( $85.4 \%, 67.1 \%, 59.8 \%$ vs $75.6 \%$, $44.6 \%, 37.3 \%, P<0.001$, respectively) also existed in patients without PVTT (Figure 4A, 4B) and in patients with single tumor (Figure 4C, 4D) (DFS: 67.6\%, 54.9\%, $52.1 \%$ vs $46.5 \%, 29.9 \%, 24.8 \%, P<0.001$, respectively; OS: $85.9 \%, 70.4 \%, 66.2 \%$ vs $71.5 \%, 46.5 \%, 39.4 \%, P<$ 0.001 , respectively). These results further demonstrated that preoperative ANRI was more sensitive than other clinical parameters to predict the prognosis of $\mathrm{HCC}$ patients, especially in different kinds of HCC subgroups whose survival is too difficult to be predicted.

\section{The prognostic value of ANRI combined with NLR for HCC after hepatectomy}

As both ANRI and NLR are neutrophil related factors, we decided to analyze the prognostic value for HCC survival of combining ANRI and NLR, the AUC value of the combination of the ANRI and NLR were 0.670 , it is more than ANRI (the AUC is 0.641) and NLR (the AUC is 0.580), and significant difference was found between them (all $P<0.05$ ). patients were divided into three groups: Group 1, NLR $\leq 2$ and ANRI $\leq 7.8$; Group 2 , patients with NLR $>2$ and ANRI $\leq 7.8$ or with $\mathrm{NLR} \leq$ 2 and ANRI > 7.8; Group 3, patients with both NLR $>2$ and ANRI $>7.8$.

The 1-, 3- and 5-year DFS rates of Group 1 (64.5\%, $54.8 \%$ and $51.6 \%$, respectively) were significantly higher than those of Group 2 (43.9\%, 28.6\% and 24.1\%,

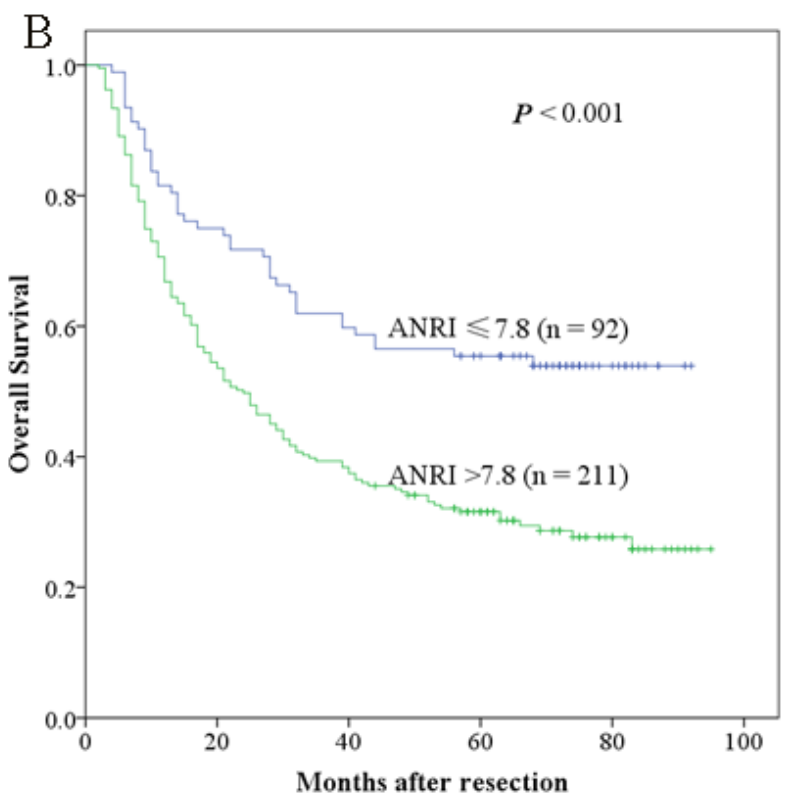

Figure 2: Relationship between ANRI and DFS/OS of HCC patients after hepatectomy. A. DFS of patients with ANRI $>$ 7.8 was significantly shorter than those with ANRI $\leq 7.8(P<0.001, \log$-rank test). B. OS of patients with ANRI $>7.8$ was also markedly shorter than those with ANRI $\leq 7.8(P<0.001$, log-rank test). 
respectively, $P<0.001)$ and Group $3(23.1 \%, 9.6 \%$ and $9.6 \%$, respectively, $P<0.001)$. Similarly, the $1-, 3-$ and 5 -year OS rates of Group 1 (83.9\%, 69.4\% and 66.1\%, respectively) were also significantly higher than those of Group 2 (72.5\%, $47.1 \%$ and $36.9 \%$, respectively, $P<0.001)$ and Group $3(51.9 \%, 15.4 \%$ and $13.5 \%$, respectively, $P<0.001$ ) (Figure 5A and 5B). Furthermore, we found that the 1-, 3- and 5-year DFS and OS rates of Group 2 were both significantly higher than those of Group $3(P<0.001$ and $P<0.001)$ ( Figure 5A and 5B).

\section{DISCUSSION}

In order to improve the outcome of HCC patients, great effort had been made on searching for valid indicators to predict $\mathrm{HCC}$ prognosis. As most $\mathrm{HCC}$ was the result of chronic liver disease, the outcome of HCC may also depends on impaired liver function secondary to the underlying pathogenic condition, rather than solely the tumor burden. Tumor Node Metastasis (TNM), which includes only pathological variables, and shows the limited prognostic value in HCC. Therefore, A reliable prognostic
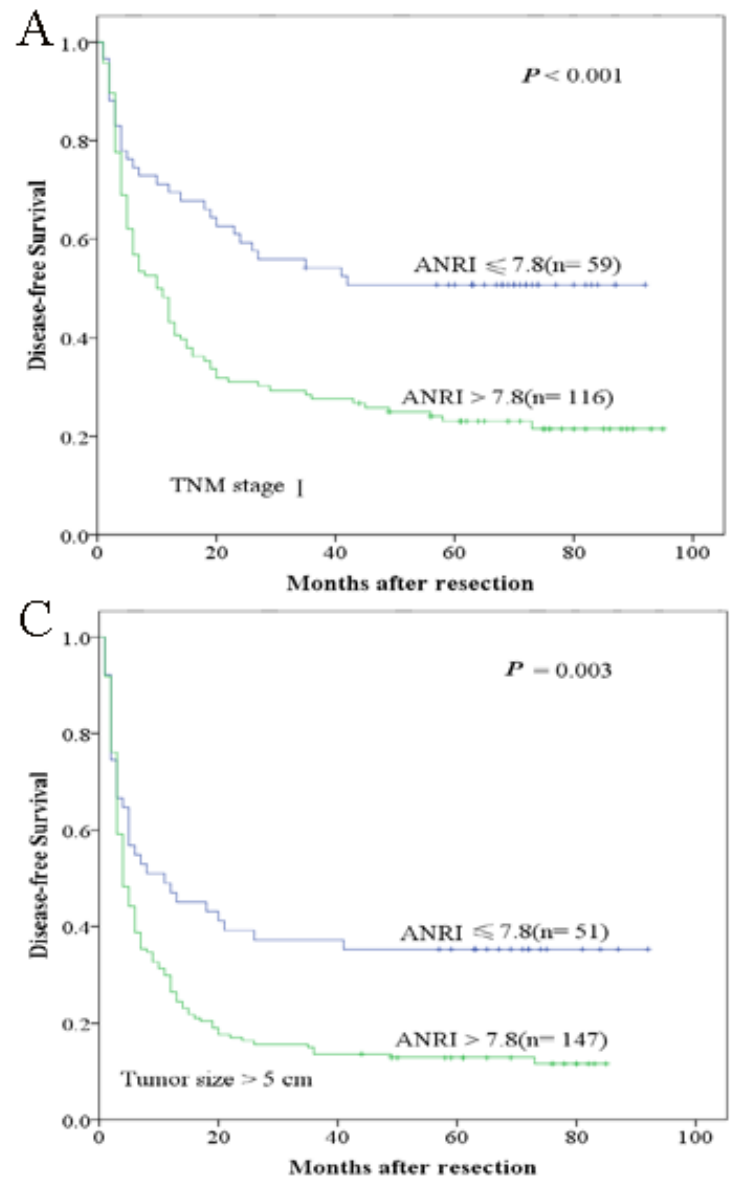

index is needed for use in routine clinical practice.

Hematological components which reflect the systemic inflammatory response have been combined to construct the inflammation-based prognostic scores to predict survival. Neutrophil plays a central role in the systemic inflammatory response, and a number of studies have demonstrated a relation between the neutrophil count or neutrophil-lymphocyte ratio and the prognosis of cancer patients [22-25], one reason of the neutrophilia is the autocrine or paraneoplastic production of myeloid growth factors, Granulocyte-colony stimulating factor (G-CSF) is one of these factors which acts selectively on bone marrow granulocytic lineage cells resulting in granulopoiesis [26-28]. Another reason may be the cancerrelated inflammation. Chronic inflammation can conduce to the initiation and progression of cancer. Because of the persistence of inflammation, the neutrophil also can directly down-regulate host cellular immunity against cancer, which affects the prognosis [14].

AST is a sensitive and reliable biochemical marker of liver injury, some liver diseases involving mitochondrial injury of hepatocytes, may lead the release of AST to
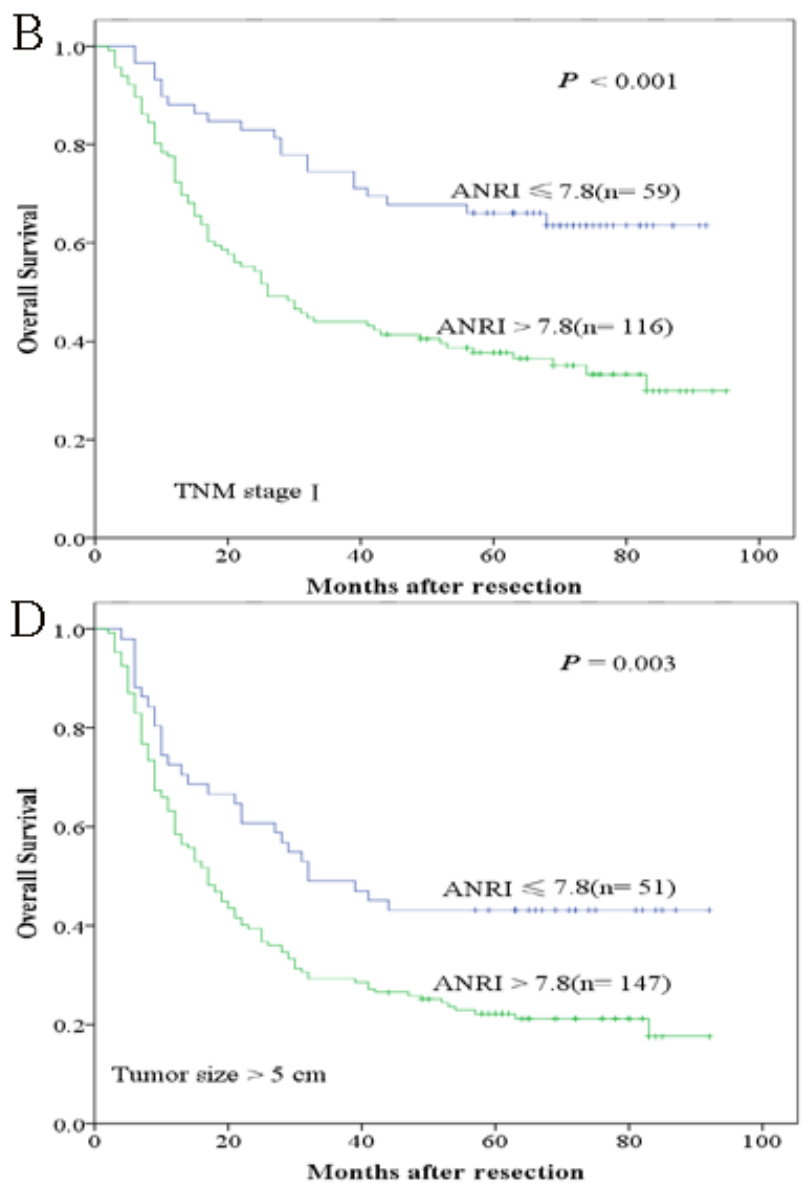

Figure 3: Kaplan-Meier survival curves of different HCC subgroups after hepatectomy. ANRI > 7.8 significantly correlated with shorter DFS and OS in subgroups with TNM stage I A., B. and tumor size $>5 \mathrm{~cm}$ C., D. 
serum. Witjes [9] had reported A higher AST level was showed to correlate with a greater influx of hepatitis B virus, which associates with decreased overall survival in HCC patients. Shen [19] and Jin [17] had certified that AST to platelet ratio index (APRI) and AST to lymphocyte ratio index (ALRI) were associated with a poor prognosis in HCC. So we aimed to demonstrate the preoperative ANRI might be a potential predictive marker for patients with HCC.

In our study, we first identified the cut-off value of preoperative ANRI according to the ROC curve, 7.8 appeared to be the optimal cut-off value of ANRI with a maximum joint sensitivity and specificity. Noteworthiness, concerning the correlationship between ANRI and clinical characteristics, we found that an elevated ANRI was positively related to $\mathrm{HBsAg}$, AST, presence of cirrhosis, tumor size and CLIP score. what's more, the c-index between ANRI and CLIP were 0.70, which showed a concordance with the CLIP score. patients having an increased levels of ANRI are more inclined to have a higher rate of PVTT and recurrence. All these data certified that ANRI could not only reflect the liver injury induced by hepatitis B but also the tumor burden and tumor progression. Using univariate analysis, we
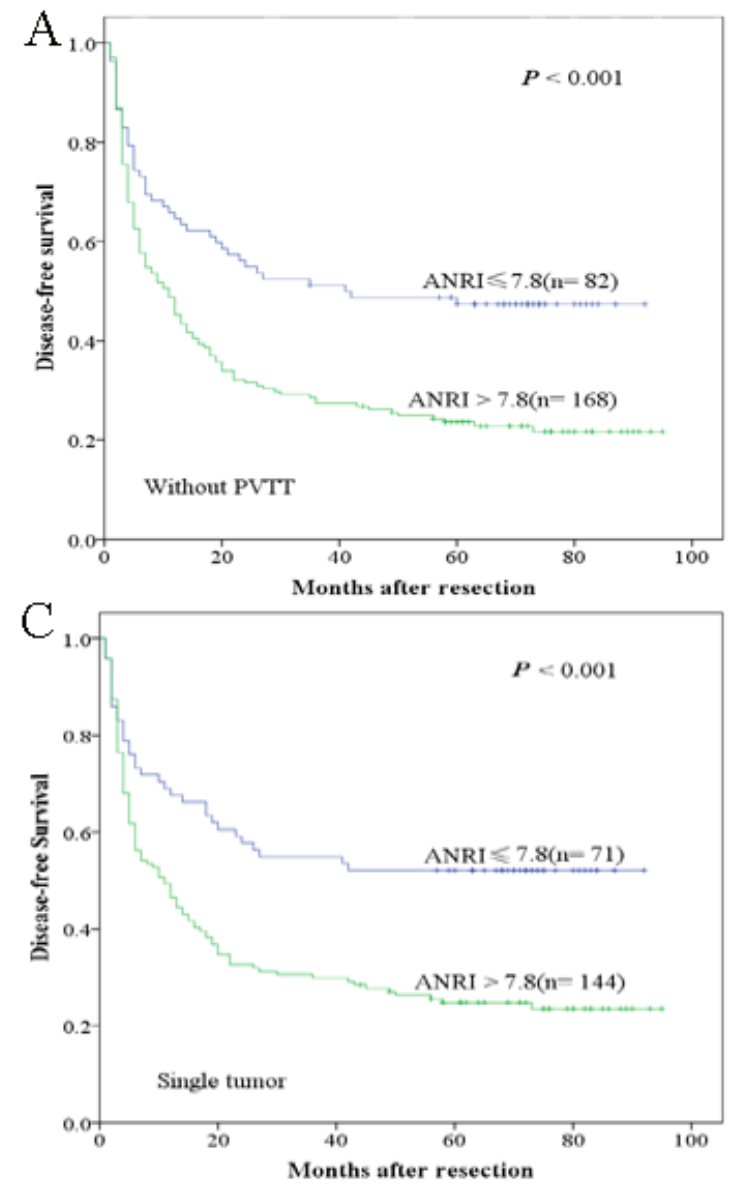

discovered many significant prognostic factors for DFS or OS of HCC, including HBsAg, gender, AFP, AST, Neutrophil, CLIP score, TNM stages, PVTT, tumor size, tumor number, poor differentiation, intraoperative blood loss, NLR, PLR, ALRI, APRI and ANRI. However, After multivariate analysis, we catch that the independent related factors for both DFS were AFP, tumor size, tumor number, PVTT, ANRI and Neutrophil. while tumor number, PVTT, ANRI, CLIP score, AST and NLR were significant independent predictors of OS. Although the AUC of ALRI is larger than ANRI, there is no statistical significance between them ( $P=0.591)$, So after multivariate analysis, ANRI was demonstrated to be superior to ALRI, APRI, NLR, PLR, which were independently correlated with DFS and OS

By a further analysis, we found that a shorter DFS and OS of HCC patients with preoperative ANRI $>7.8$ had been documented in this study. The 1-, 3- and 5-year DFS rates and OS rates of patients with high level of ANRI were markedly lower than the low level group. Shau etc [29-31] had discovered that the neutrophils suppressed the cytolytic activity of lymphocytes and natural killer cells and the degree of suppression was in proportion to the number of neutrophils. $\mathrm{CD}^{+} \mathrm{T}$ lymphocyte cells
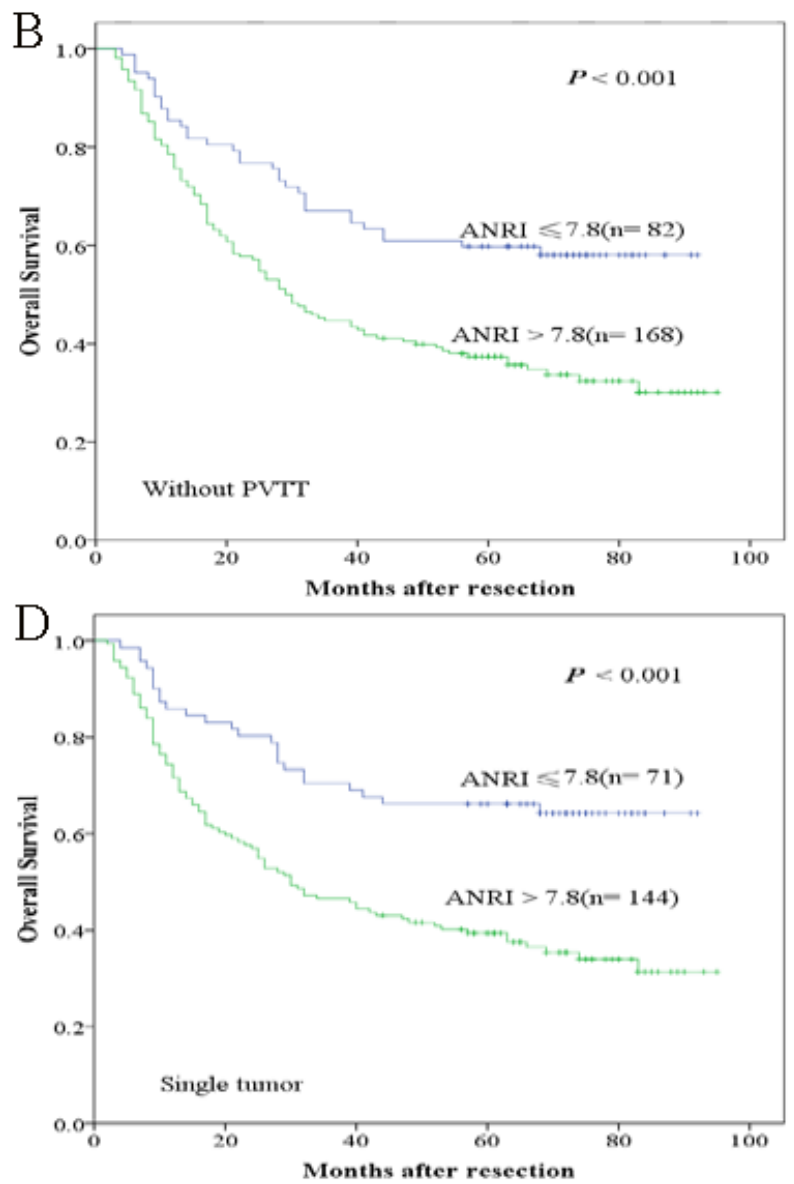

Figure 4: Kaplan-Meier survival curves of different HCC subgroups after hepatectomy. ANRI $>7.8$ significantly correlated with shorter DFS and OS in patients without PVTT A., B. and with single tumor C., D. 
act as a sensor in detecting precancerous cells and then regulate their eradication [32], these can prevent the occurrence and development of $\mathrm{HCC}$. The loss of $\mathrm{CD}^{+}$ $\mathrm{T}$ lymphocyte accompanied with the impaired activation of $\mathrm{CD}^{+} \mathrm{T}$ lymphocyte cells may cause the insufficient secretion of cytotoxin performing anti-carcinogenic function in the neoplastic microenvironments [33], what's more, Neutrophils potentiate cancer cell migration, invasion, and dissemination by secreting immunoreactive molecules such as hepatocyte growth factor (HGF) [34, 35], oncostatin M [36], b2-integrins [37] or neutrophil elastase [38]. With the progression of tumor diseases, the hepatic parenchymal cells are damaged, intracellular AST will be released into the blood which will cause a high concentration of serum AST. Therefore, the elevated preoperative ANRI indicates poorer prognosis of HCC.

In order to explicit the the prognostic value of preoperative ANRI in different subgroups of HCC patients, we found that preoperative ANRI had significant prognostic value for both DFS and OS in patients with TNM stage I, that is to say preoperative ANRI could be used to predict recurrence in early HCC. Besides, in subgroup with tumor size $>5 \mathrm{~cm}$, patients with single tumor or patients without PVTT, preoperative ANRI > 7.8 also show its prognostic value in predicting poorer DFS and OS, all of these provided further evidence that preoperative ANRI can act as a potential prognostic marker to predict survival in HCC patients after hepatectomy.

Because both NLR and ANRI are prognostic factors relating neutrophil, we tried to explore whether the prognostic value could be expanded by the combination of them. Interestingly, our result exhibited that the combination of ANRI and NLR had a better prognostic

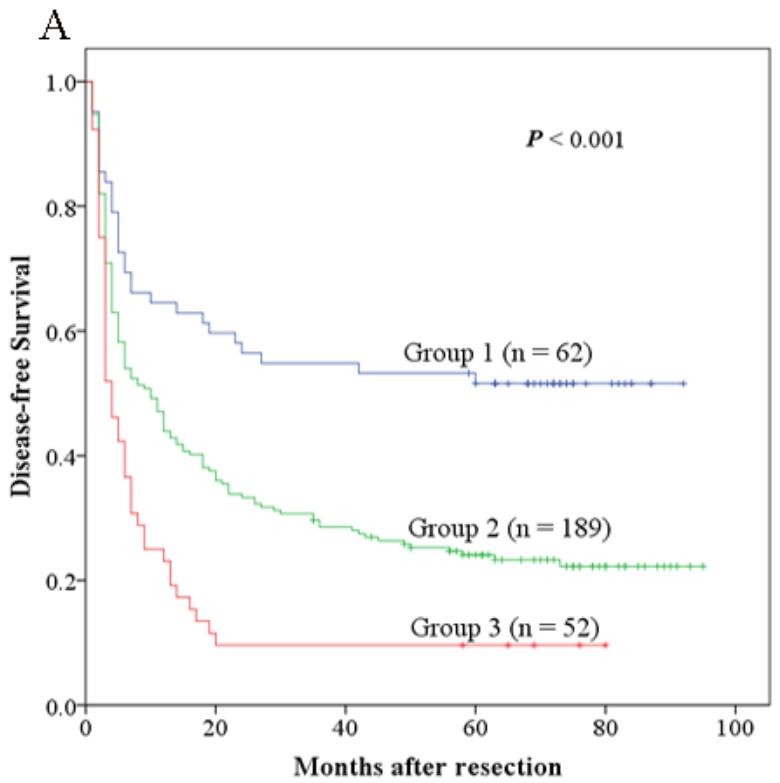

value than either one alone, patients with NLR $\leq 2$ and ANRI $\leq 7.8$ had the best DFS and OS rates, patients with NLR $>2$ and ANRI $\leq 7.8$ or with NLR $\leq 2$ and ANRI $>$ 7.8 were the second, then patients with both NLR $>2$ and ANRI $>7.8$ were the worst.

As for other HCC biomarkers such as AFP, Glypican-3 (GPC3). the diagnostic sensitivity and accuracy haven't been conformed. Bialecki [39] had reported that $30 \%$ of HCC patients didn't have a remarkable rise of serum AFP, and AFP may also increase initially in the early stages of HCC but then drop or even normalize before rising again though disease progression occurs [40], Additionally, patients with chronic hepatitis and liver cirrhosis may have increased levels of AFP without HCC [41]. GPC3 is a member of the heparin sulfate proteoglycans, it can be secreted from $\mathrm{HCC}$ cells, However, it can be detected in only $40-53 \%$ of HCC patients and 33\% of HCC patients seronegative for AFP [41-43]. Childs-pugh is a marker reflecting the liver function. For many HCC patients, their liver function is Childs pugh A, or liver function is normal. preoperative liver function may predict the short-term prognosis, but not for the long-term prognosis, because the liver function may change daily. And CLIP is a score combining factors of tumor and liver function, but it didn't display significance in DFS after multivariate analysis. So the ANRI not only meets the demands of highly precise diagnosis and prognosis, but also reduces patients' economic cost because it deduced from the routine examination, not extra examination. However, there are still some deficiencies in the present study. First, it is a single-institution, retrospective study. Second, our database mainly based on a cohort of HCC patients

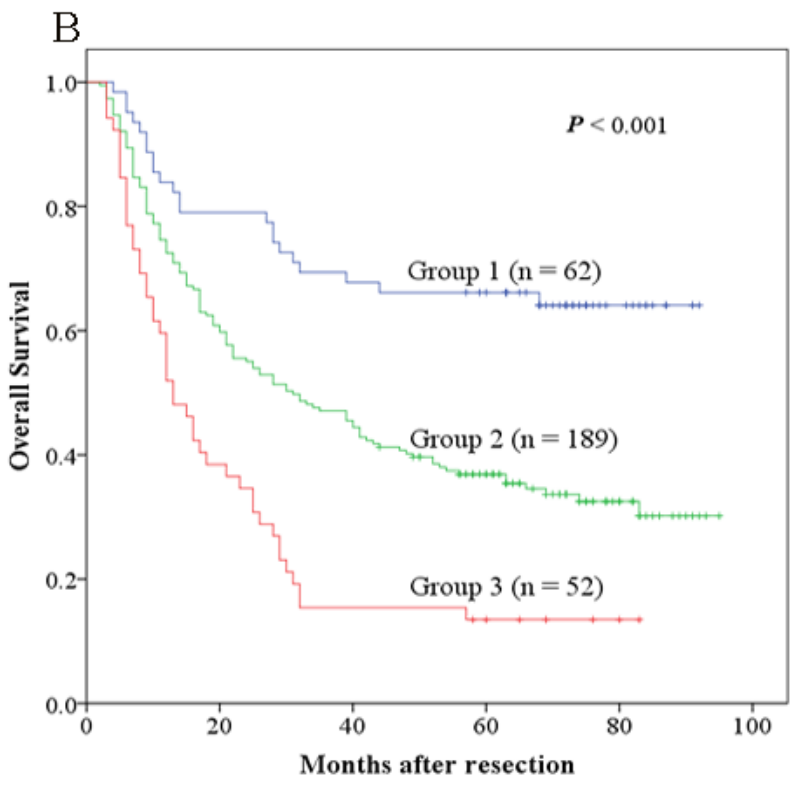

Figure 5: The combination of ANRI and NLR was found to enhance prognostic accuracy for HCC. Disease-free survival curves A. and overall survival curves B. Group1: both ANRI $\leq 7.8$ and NLR $\leq 2$; Group2: both ANRI $\leq 7.8$ and NLR $>2$ or both ANRI $>$ 7.8 and NLR $\leq 2$; Group3: both ANRI $>7.8$ and NLR $>2$. 
with predominant HBV infection in China. Therefore, the results for application in Western populations with predominant HCV infection or a history of alcohol abuse should be further confirmed.

In conclusion, our study demonstrate that the elevation of preoperative ANRI can be used as a prognostic factor for predicting the prognosis of patients with HCC after hepatectomy. These findings may suggest us to make the treatment plan considering not only TNM stage but also these prognosis related serum biomarkers. Only in this way can we acquire better personalised therapy for patients with HCC. In the future, this simple preoperative prognostic evaluation could be used to screen patients for personalized therapy.

\section{MATERIALS AND METHODS}

\section{Ethics statement}

Written informed consent was provided to all patients prior to surgery. Study approval was granted by the independent ethics committees at the First Affiliated Hospital of Sun Yat-sen University. This study was conducted in accordance with the ethical standards of the World Medical Association Declaration of Helsinki.

\section{Study population}

A total of 303 histologically proven HCC patients with hepatic resection from our hospital were recruited between July 2006 and December 2009. Routine assessment was performed within 7 days before surgery, including a complete physical examination, hematologic and biochemistry profiles, chest X-ray, abdominal ultrasound and computed tomography (CT) or magnetic resonance imaging (MRI).

The eligibility criteria contained: the International Union Against Cancer (seventh edition) TNM stage I, II, IIIA or IIIB; Child-Pugh class A hepatic function; age 18-80 years. Exclusion criteria contained: TNM stage IIIC, IV; existing second malignancy or history of second malignancy within the past five years; hematologic disorders; systematic inflammatory diseases; perioperative dysfunction of vital organs; or percutaneous ablation, transcatheter arterial chemoembolization (TACE), chemotherapy or radiotherapy within one month after surgery.

\section{Treatment and follow-up}

Hepatectomy was defined as radical when there was no evidence of distant metastases and tumor clearance was complete both macroscopically and histologically.
All 303 patients were regularly followed up according to institutional practice, including liver ultrasound, chest X-ray and serum AFP every three months, and contrast CT every 6 months. Tumor relapse was diagnosed by clinical, radiological and/or pathological test. The mean for the postoperative follow-up period was 30.0 months (range, 2.0 to 95.0 months). Disease-free survival (DFS) was calculated from the date of surgery to the date of recurrence, and overall survival (OS) from the date of surgery to the date of HCC-associated death.

\section{Definition}

Five inflammatory factors, including NLR, PLR, ALRI, APRI and ANRI were included in this analysis. The definitions of these factors are as follows: NLR = Neutrophil count/lymphocyte count; PLR = platelet count / lymphocyte count; ALRI = (AST value/ lymphocyte count $) \times 10^{9} / \mathrm{U} ;$ APRI $=($ AST value $/$ platelet count $) \times$ $10^{9} / \mathrm{U} ; \mathrm{ANRI}=($ AST value $/$ Neutrophil count $) \times 10^{9} / \mathrm{U}$. Postoperative complications were defined as occurrence of any medical or surgical complication during the hospital stay, such as Liver failure/insufficiency, Bile leak, Biloma/ abscess, Intra-abdominal infection, Hemorrhage, Ascites, Wound infection/dehiscence, Pleural effusion, Pneumonia, Atelectasis, Arrhythmia, Heart failure, Urinary retention, Urinary tract infection, Renal insufficiency/failure, Ileus and Delayed Gastric Emptying.

\section{Statistical analysis}

Statistical analysis was performed using SPSS for Windows version 20.0 (SPSS, Chicago, IL, USA). Receiver operating characteristic (ROC) curve analysis was performed to select the most appropriate cut-off values for ANRI to stratify patients at a high risk of death. The pROC package of R software (version 3.0.1) was used to evaluate the significance for the difference of area under the ROC curve (AUC). The $\chi 2$ test was used to compare categorical variables, and correlationship between variables were detected by Pearson test. The Kaplan-Meier method was used to estimate the survival rates for different groups, and the equivalences of the survival curves were tested by log-rank statistics. The Cox proportional hazards model was used for univariate and multivariate survival analyses. $P<0.05$ was considered statistically significant.

\section{Abbreviations}

HCC: hepatocellular cancer; TNM: Tumor Node Metastasis; NLR: neutrophil/lymphocyte ratio index; PLR: platelet/lymphocyte ratio index; AST: aspartate aminotransferase ratio index; ALRI: aspartate aminotransferase/lymphocyte count ratio index; APRI: 
aspartate aminotransferase/ platelet count ratio index; ANRI: aspartate aminotransferase/ neutrophil count ratio index; CLIP: cancer of the liver Italian program; GPC3: Glypican-3; CT: computed tomography; MRI: magnetic resonance imaging; TACE: transcatheter arterial chemoembolization; PLT: platelet; AFP:alpha-fetoprotein; ROC: receiver operating characteristics; DFS: disease-free survival; OS: overall survival; HBsAg: hepatitis B surface antigen; PVTT: portal vein tumor thrombus; TBIL: total bilirubin.

\section{ACKNOWLEDGMENTS}

This study was supported by the National High Technology Research and Development Program of China (863 Program) (2012AA021007 \&2012AA021008), the Research Fund for the Doctoral Program of Higher Education of China by Ministry of Education (20110171120077), the National Natural Science Foundation of China (81201918, 81373156 and 81471583), the Special Fund for Science Research by Ministry of Health (201302009), the Key Clinical Specialty Construction Project of National Health and Family Planning Commission of the People's Republic of China, the Guangdong Provincial Key Laboratory Construction Projection on Organ Donation and Transplant Immunology (2013A061401007), Guangdong Provincial international Cooperation Base of Science and Technology (Organ Transplantation) (2015B050501002), Pearl River Nova Program of Guangzhou (201506010014), and Guangdong Provincial Natural Science Funds for Distinguished Young Scholars (2015A030306025). Science and Technology Project of Guangdong Province (2012B031800099), the Scientific Research Foundation for the Returned Overseas Chinese Scholars, State Education Ministry (2015, NO.311). The funders had no role in study design, data collection and analysis, decision to publish, or preparation of the manuscript.

\section{CONFLICTS OF INTEREST}

There is no conflict of interest.

\section{REFERENCES}

1. El-Serag HB, Rudolph KL. Hepatocellular carcinoma: epidemiology and molecular carcinogenesis. Gastroenterology. 2007; 132:2557-2576.

2. Ji F, Fu SJ, Shen SL, Zhang LJ, Cao QH, Li SQ, Peng BG, Liang LJ, Hua YP. The prognostic value of combined TGF- $\beta 1$ and ELF in hepatocellular carcinoma. BMC cancer. 2015; 15:116.

3. Ng KK, Lo CM, Liu CL, Poon RT, Chan SC, Fan ST. Survival analysis of patients with transplantable recurrent hepatocellular carcinoma: implications for salvage liver transplant. Arch Surg. 2008; 143: 68-74.

4. Kim DY, Paik YH, Ahn SH, Youn YJ, Choi JW, Kim JK, Lee KS, Chon CY, Han KH. PIVKA-II is a useful tumor marker for recurrent hepatocellular carcinoma after surgical resection. Oncology. 2007; 72:52-57.

5. Jemal A, Bray F, Center MM, Ferlay J, Ward E, Forman D. Global cancer statistics. CA Cancer J Clin. 2011; 61:69-90.

6. Kim HC, Nam CM, Jee SH, Kan KH, Oh DK, Suh I. Normal serum aminotransferase concentration and risk of mortality from liver diseases: prospective cohort study. BMJ. 2004; 328:98.

7. van Beek JH, de Moor MH, de Geus EJ, Lubke GH, Vink JM, Willemsen G, Boomsma DI. The genetic architecture of liver enzyme levels: GGT, ALT and AST. Behav Genet. 2013; 43:329-339.

8. Lee TH, Kim WR, Benson JT, Therneau TM, Melton LJ 3rd. Serum aminotransferase activity and mortality risk in a United States community. Hepatology. 2008; 47:880-887.

9. Witjes CD, IJzermans JN, van der Eijk AA, Hansen BE, Verhoef C, de Man RA. Quantitative HBV DNA and AST are strong predictors for survival after $\mathrm{HCC}$ detection in chronic HBV patients. Neth J Med. 2011; 69:508-513.

10. Fu SJ, Shen SL, Li SQ, Hua YP, Hu WJ, Liang LJ, Peng BG. Prognostic value of preoperative peripheral neutrophilto-lymphocyte ratio in patients with HBVassociated hepatocellular carcinoma after radical hepatectomy. Med Oncol. 2013; 30:721.

11. Balkwill F, Mantovani A. Inflammation and cancer: back to Virchow? Lancet. 2001; 357:539-545.

12. Coussens LM, Werb Z. Inflammation and cancer. Nature. 2002; 420:860-867.

13. Mantovani A, Allavena P, Sica A, Balkwill F. Cancerrelated inflammation. Nature 2008; 454:436-444.

14. Teramukai S, Kitano T, Kishida Y, Kawahara M, Kubota K, Komuta K, Minato K, Mio T, Fujita Y, Yonei T, Nakano K, Tsuboi M, Shibata K, et al. Pretreatment neutrophil count as an independent prognostic factor in advanced non-smallcell lung cancer: an analysis of Japan Multinational Trial Organisation LC00-03. Eur J Cancer. 2009; 45:1950-1958.

15. Wilcox RA, Ristow K, Habermann TM, Inwards DJ, Micallef IN, Johnston PB, Colgan JP, Nowakowski GS, Ansell SM, Witzig TE, Markovic SN, Porrata L. The absolute monocyte and lymphocyte prognostic score predicts survival and identifies high-risk patients indiffuse large-B-cell lymphoma. Leukemia. 2011; 25:1502-9.

16. He JR, Shen GP, Ren ZF, Qin H, Cui C, Zhang Y, Zeng YX, Jia WH. Pretreatment levels of peripheral neutrophils and lymphocytes as independent prognostic factors in patientswith nasopharyngeal carcinoma. Head Neck. 2012; 34:1 769-76.

17. Jin J, Zhu P, Liao Y, Li J, Liao W, He S. Elevated preoperative aspartate aminotransferase to lymphocyte ratio index as an independent prognostic factor for patients with hepatocellular carcinoma after hepatic resection. 
Oncotarget. 2015; 6:19217-19227. doi: 10.18632/ oncotarget.4265.

18. Yang Z, Zhang J, Lu Y, Xu Q, Tang B, Wang Q, Zhang W, Chen S, Lu L, Chen X. Aspartate aminotransferaselymphocyte ratio index and systemic immuneinflammation index predict overall survival in HBV-related hepatocellular carcinoma patients after transcatheter arterial chemoembolization. Oncotarget. 2015; 6:43090-43098. doi: 10.18632/oncotarget.5719.

19. Shen SL, Fu SJ, Chen B, Kuang M, Li SQ, Hua YP, Liang LJ, Guo P, Hao Y, Peng BG. Preoperative Aspartate Aminotransferase to Platelet Ratio is an Independent Prognostic Factor for Hepatitis B-Induced Hepatocellular Carcinoma After Hepatic Resection. Ann Surg Oncol. 2014; 21:3802-3809.

20. Edmondson HA, Steiner PE. Primary carcinoma of the liver: a study of 100 cases among 48,900 necropsies. Cancer. 1954; 7:462-503.

21. Sobin LH, Gospodarowicz MK, Wittekind C. International Union Against Cancer (UICC): TNM classification of malignant tumours. 7th edn. New York: Wiley-Liss; 2010.

22. Huang GQ, Zhu GQ, Liu YL, Wang LR, Braddock M, Zheng MH, Zhou MT. Stratified neutrophil-tolymphocyte ratio accurately predict mortality risk in hepatocellular carcinoma patients following curative liver resection. Oncotarget. 2016; 7:5429-39. doi: 10.18632/ oncotarget.6707.

23. Gao F, Li X, Geng M, Ye X, Liu H, Liu Y, Wan G, Wang X. Pretreatment neutrophil-lymphocyte ratio: an independent predictor of survival in patients with hepatocellular carcinoma. Medicine (Baltimore). 2015; 94:e639.

24. Nakashima H, Matsuoka Y, Yoshida R, Nagata M, Hirosue A, Kawahara K, Sakata J, Arita H, Hiraki A, Nakayama H. Pre-treatment neutrophil to lymphocyte ratio predicts the chemoradiotherapy outcome and survival in patients with oral squamous cell carcinoma: a retrospective study. BMC Cancer. 2016; 16:41.

25. Grenader T, Waddell T, Peckitt C, Oates J, Starling N, Cunningham D, Bridgewater J. Prognostic value of neutrophil to lymphocyte ratio in advanced oesophagogastric cancer: exploratory analysis of the REAL-2 trial. Ann Oncol. 2016; pii: mdw012.

26. Teramukai S, Kitano T, Kishida Y, Kawahara M, Kubota K, Komuta K, Minato K, Mio T, Fujita Y, Yonei T, Nakano K, Tsuboi M, Shibata K,et al. Pretreatment neutrophil count as an independent prognostic factor in advanced non-smallcell lung cancer: An analysis of Japan Multinational Trial Organisation LC00-03. Eur J Cancer. 2009; 45:1950-1958.

27. Tachibana M, Miyakawa A, Tazaki H, Nakamura K, Kubo A, Hata J, Nishi T, Amano Y. Autocrine growth of transitional cell carcinoma of the bladder induced by granulocyte-colony stimulating factor. Cancer Res. 1995; 55:3438-3443.

28. Oshika Y, Nakamura M, Abe Y, Fukuchi Y, Yoshimura M,
Itoh M, Ohnishi Y, Tokunaga T, Fukushima Y, Hatanaka H, Kijima H, Yamazaki H, Tamaoki N, et al. Growth stimulation of non-small cell lung cancer xenografts by granulocytemacrophage colony-stimulating factor (GMCSF). Eur J Cancer 1998; 34:1958-1961.

29. Petrie HT, Klassen LW, Kay HD. Inhibition of human cytotoxic $\mathrm{T}$ lymphocyte activity in vitro by autologous peripheral blood granulocytes. J Immunol. 1985; 134:230234.

30. el-Hag A, Clark RA. Immunosuppression by activated human neutrophils. Dependence on the myeloperoxydase system. J Immunol.1987; 139:2406-2413.

31. Shau HY, Kim A. Suppression of lymphokine-activated killer induction by neutrophils. J Immunol. 1988; 141:43954402.

32. Kang TW, Yevsa T, Woller N, Hoenicke L, Wuestefeld T, Dauch D, Hohmeyer A, Gereke M, Rudalska R, Potapova A, Iken M, Vucur M, Weiss S, et al. Senescence surveillance of pre-malignant hepatocytes limits liver cancer development. Nature. 2011; 479:547-551.

33. Witkowski M, Spangenberg HC, Neumann-Haefelin C, Büttner N, Breous E, Kersting N, Drognitz O, Hopt UT, Blum HE, Semmo N, Thimme R. Lack of ex vivo peripheral and intrahepatic alpha-fetoprotein-specific CD4+ responses in hepatocellular carcinoma. Int J Cancer. 2011; 129:21712182.

34. Wislez M, Rabbe N, Marchal J, Milleron B, Crestani B, Mayaud C, Antoine M, Soler P, Cadranel J. Hepatocyte growth factor production by neutrophils infiltrating bronchioloalveolar subtype pulmonary adenocarcinoma: role in tumor progression and death. Cancer Res. 2003; 63:1405-1141.

35. Queen MM, Ryan RE, Holzer RG, Keller-Peck CR, Jorcyk CL. Breast cancer cells stimulate neutrophils to produce oncostatin M: potential implications for tumor progression. Cancer Res. 2005; 65:8896-8904.

36. Li YW, Qiu SJ, Fan J, Zhou J, Gao Q, Xiao YS, Xu YF. Intratumoral neutrophils: A poor prognostic factor for hepatocellular carcinoma following resection. J Hepatol. 2011; 54:497-505.

37. Strell C, Lang K, Niggemann B, Zaenker KS, Entschladen F. Neutrophil granulocytes promote the migratory activity of MDA-MB-468 human breast carcinoma cells via ICAM1. Exp Cell Res. 2010; 316:138-148.

38. Houghton AM, Rzymkiewicz DM, Ji H, Gregory AD, Egea EE, Metz HE, Stolz DB, Land SR, Marconcini LA, Kliment CR, Jenkins KM, Beaulieu KA, Mouded M, et al. Neutrophil elastase-mediated degradation of IRS-1 accelerates lung tumor growth. Nat Med. 2010; 16:219-223.

39. Bialecki ES, Di Bisceglie AM. Diagnosis of hepatocellular carcinoma. HPB. 2005; 7:26-34.

40. Chen DS, Sung JL, Sheu JC, Lai MY, How SW, Hsu HC, Lee CS, Wei TC. Serum $\alpha$-fetoprotein in the early stage of human hepatocellular carcinoma. Gastroenterology. 1984; 
86:1404-1409.

41. Shiota G, Miura N. Biomarkers for hepatocellular carcinoma. Clin J Gastroenterol. 2012; 5:177-182.

42. Capurro M, Wanless IR, Sherman M, Deboer G, Shi W, Miyoshi E, Filmus J, Glypican-3: a novel serum and histochemical marker for hepatocellular carcinoma. Gastroenterology. 2003; 125:89-97.

43. Nakatsura T, Yoshitake Y, Senju S, Monji M, Komori H, Motomura Y, Hosaka S, Beppu T, Ishiko T, Kamohara H, Ashihara H, Katagiri T, Furukawa Y, Fujiyama S, Ogawa M, Nakamura Y, Nishimura Y. Glypican-3, overexpressed specifically in human hepatocellular carcinoma, is a novel tumor marker. Biochem Biophys Res Commun. 2003; 306:16-25. 